\title{
Planning with PlaceMaker: complex indices for sustainable projects
}

\author{
M. Sepe \\ C.N.R .- DPU Università di Napoli Federico II, Naples, Italy
}

\begin{abstract}
Questions connected to urban analysis study aspects that are not univocally translatable into objective facts with particular regard to three issues: the scientificity, and so the objectivity of the results, and the repeatability of the method in different contexts; the updating capability, and so the possibility of adding new data, to modify the existing data and to obtain other results; the times, and so the sustainable possibility of using the results with respect to the evolution of a sustainable programming and town planning process.

To answer these questions the method of analysis called PlaceMaker and the associated software, actually under development, have the following main characteristics: flexibility, facility and rapidity of use, a strong graphical impact, and indexing of the results. PlaceMaker is a method for analysing the contemporary urban landscape that is designed to identify the elements that do not feature in traditional mapping and which constitute the contemporary identity of the places, representing them in a complex map that renders those places intelligible. Starting from these premises, the aim of this work, carried out in the framework of a Convention between Consiglio Nazionale delle Ricerche and Dipartimento di Progettazione Urbana - Università di Napoli Federico II, is to investigate the construction of complex indices starting from data and symbols related to the place-identity. In order to support the study and the project of the sustainable urban landscapes, the symbols created for the elaboration of the complex map, the final result of the analysis, are translated by the PlaceMaker software into numerical indices. Owing to these characteristics, the prime users of PlaceMaker are urban planners, administrators, citizens and all people involved in construction of a sustainable city. To complete the work suitable case studies are presented.
\end{abstract}

Keywords: placemaking, sustainable urban planning, multimedia software, complex indices, sustainable place-identity index. 


\section{Introduction}

The quality of a place in a city does not merely depend on the city's physical resources but also on subjective factors. Most of the latter are determined by the interaction of the patterns constituting the city as a whole with the significance given by users to each of the places they encounter and exploit. Current studies on indicators of urban sustainability tend to focus above all on such factors as population, water, air, energy, noise, transport and urban vegetation $[1,2]$. Attention is rarely paid to the urban image, and if it is, this is chiefly in relation to the aesthetic impact, as a factor in calculating urban sustainability [3]. Starting from these premises, this study, carried out in the framework of a convention between the Consiglio Nazionale delle Ricerche and Dipartimento di Progettazione Urbana - Università di Napoli Federico II, sets out to investigate the possibility of calculating the sustainability of urban identity using what we have called the sustainable place-identity index. The characteristic features of a place's identity are established using PlaceMaker, a method for analysing the urban landscape designed to identify elements that do not feature in traditional mapping and which constitute the contemporary identity of the places, representing them in a complex map that renders the place intelligible [4]. This method assembles, elaborates and reconstructs the data deriving from surveys based on physical reconnaissance, sensory perceptions, graphical elaboration, and photographic and video records. It sets this data against an overview of expectations, analysis of traditional cartography and a questionnaire given to local inhabitants. Specific software is currently being developed to connect up and communicate the information contained in the complex map. This will support PlaceMaker in all its phases and the creation of the map. PlaceMaker makes it possible to represent and interpret places in a territory by drawing up interactive maps, using symbols and elements connected to multimedia schedules that can be continuously updated. The main characteristics of PlaceMaker are: flexibility, ease and rapidity of use, strong graphical impact, indexing of results. In order to render the results of the interpretation objective and useful for sustainable urban planning, the software links the symbols of the complex map to numerical indices, making it possible to calculate the quality, potentiality and weaknesses of the places represented in the map. We put the sustainable place-identity index and its potential in studying sustainable urban landscapes to the test using two case studies based on historical city centres. Both places, one in Italy and the other in Japan, were destroyed by violent earthquakes and suffered wide-scale damage. This article is organized as follows: section 2 outlines the proposed index; section 3 illustrates the two case studies and relative observations; and in section 4 we draw the conclusions.

\section{The sustainable place-identity index}

Establishing the identity of a site located in a certain territory is the key to a comprehensive interpretation of place, showing its significance and value with respect to safeguarding, on the one hand, and planning and/or reconstruction, on 
the other. The peculiarity of an urban site consists in the concentration of buildings, inhabitants or activities which characterises it. These elements may create critical situations and congestion, above all in terms of environmental sustainability.

Studies concerning indicators of urban sustainability rarely refer to urban image. They generally focus on the parameters of population, water, air, energy, noise, transport and urban vegetation. The complexity of the contemporary city makes it very difficult for citizens to arrive at interpretations and value judgements, for they are invariably confronted with new urban features which cannot be fully decoded. The identity of a place is an element which, while comprising the sum of many individual features, is a comprehensive value, which can nonetheless be arrived at by measuring its constituent elements. Determining the sustainability of place identity involves monitoring the elements which go to make up urban identity seen in the perspective of sustainability. We believe that the creation of a complex index generating numerical values may be useful input for the process of sustainable urban construction and transformation. In order to study the sustainability of a place by means of data concerning urban identity, we shall use what we have called the sustainable place-identity index.

The method of analysis used to identify the characteristic features of place identity is provided by PlaceMaker. The index is characterised above all by referring to the context and its phenomena, and by its flexibility, ease of updating and compatibility with planning tools. It is defined by the value of a set of elements expressed as symbols shown on the complex map drawn up using the PlaceMaker. Each symbol is associated with an element of place, to which is attributed a value of integral weight $\mathrm{w}_{\mathrm{i}}$ ranging from 1 to 5 . This weight is determined by the typology of the indicator to be calculated. The lowest value of integral weight $\mathrm{wi}_{\mathrm{i}}=1$ has little impact in calculating the index, while the highest value $\mathrm{w}_{\mathrm{i}}=5$ counts for a lot. The overall number of elements is taken to be $n$, so that $i \in\{1, \ldots, n\}$. Each element of the complex map is defined by a set of features (varying in typology and number from one element to another), each having its own integral value $c_{i j}$ ranging from 1 to 5 . Thus $c_{i j}$ denotes the feature $\mathrm{j}$ of the element $\mathrm{i}$ and $\mathrm{j} \in\left\{1, \ldots, \mathrm{m}_{\mathrm{i}}\right\}$, where mi represents the total number of features of the element $i$. All the elements denote the quantitative presence of that element in the place. The value $v_{i}$ of an element is given by the median value of its features, calculated thus:

$$
\mathrm{v}_{\mathrm{i}}=\frac{1}{\mathrm{~m}_{\mathrm{i}}} \sum_{\mathrm{j}=1}^{\mathrm{m}_{\mathrm{i}}} \mathrm{c}_{\mathrm{ij}} .
$$

It is possible to define an absolute index $\mathrm{I}_{\mathrm{a}}$ and a median index $\mathrm{Im}$. The absolute index is calculated thus:

$$
\mathrm{I}_{\mathrm{a}}=\sum_{\mathrm{i}=1}^{\mathrm{n}} \mathrm{WiVi}_{\mathrm{i}},
$$

and corresponds to the weighted sum of the value of all the elements of a place. This value in this index depends on both the quantity and the quality of the elements present. The median index $\mathrm{Im}_{\mathrm{m}}$ is calculated thus: 


$$
\mathrm{Im}_{\mathrm{m}}=\mathrm{I}_{\mathrm{a}} / \mathrm{n}
$$

and depends only on the quality of the elements present.

\section{The case studies of the historical centres of Sant'Angelo dei Lombardi, Southern Italy, and Kobe, Southern Japan}

In order to try out this index and its potential in enhancing cultural resources and sustainable planning in cities, we calculated the indices for the historical centres of Sant'Angelo dei Lombardi in Irpinia (Southern Italy) and Kitano-Cho at Kobe (Southern Japan). The historical centres of Sant'Angelo dei Lombardi and Kobe were destroyed in 1980 and 1995 respectively by earthquakes measuring 6.9 and 7.3 on the Richter scale. The casualties ran into millions and there was incalculable material damage as well as a grave impact on place identity and collective memory. The culture and traditions of the populations in the two historical centres are clearly very different. Nonetheless the two sites can be compared in terms not only of the earthquake but also of surface area, history, orography, the typology of reconstruction based on how it was and where it was and some perceptions we shall illustrate below.

\subsection{The sustainable place-identity index of Sant'Angelo dei Lombardi}

The complex map of the historical centre of Sant'Angelo dei Lombardi, fig. 1, shows how the historical centre interacts powerfully with the rest of the town, in spite of there being no real continuity in the architectonic approach. The lay-out is concentric, with all the streets linking up and feeding into one another. The characteristic structure of a hilltop village was not seriously impaired during the reconstruction. Buildings rarely exceed two storeys, and have pitched roofs with virtually no decoration. The impression created by the historical centre is of a good level of maintenance, carried out recently and with a pleasant colour scheme, but there appear to be few residents. One detects few of the elements denoting globalisation, apart from the satellite dishes on some houses and the television mast. The urban décor is well thought out, particularly in Piazza De Sanctis, where the paving reflects the various itineraries in the use of different sizes of porphyry slabs and cubes, variously oriented, and also alternative materials. Some elements are immediately apparent: the silence pervading the various places, occasionally broken by voices, passing cars or work on building sites; the sound of the wind, particularly appreciable in the open areas; occasional passers by, invariably elderly people or a few youngsters, above all near the bars in Piazza De Sanctis; in spite of the play area near the castle, no children were encountered. The view over the surrounding countryside is a constant element of perception, evoking the difficult relationship with the forces of nature. The castle and the cathedral are two of the major historical monuments; the castle in particular can be seen from virtually everywhere in the town. There is little stimulus for perceptions of smell and taste. The only public transport is the extra-urban bus services. There are no venues for aggregation, 
and in fact there seems to be little life in the town; there are no shops in the historical centre, apart from one boutique dealing in hand-made crib scenes on the ramp of Piazza De Sanctis. The few people whom we interviewed did not seem to want to remember the earthquake. They declared that there are no particularly interesting monuments, that everything has gone back to being as it was before the catastrophe, and that they are happy with the state of the historical centre. Many monuments have undergone a change of function, including the town hall and the prison. Memories of the earthquake seem to have been deliberately dismissed: it is physically present only in the monument to the earthquake victims in Via Mancini, and in the ruins still standing near the statue of the Madonna in Piazza Umberto I.

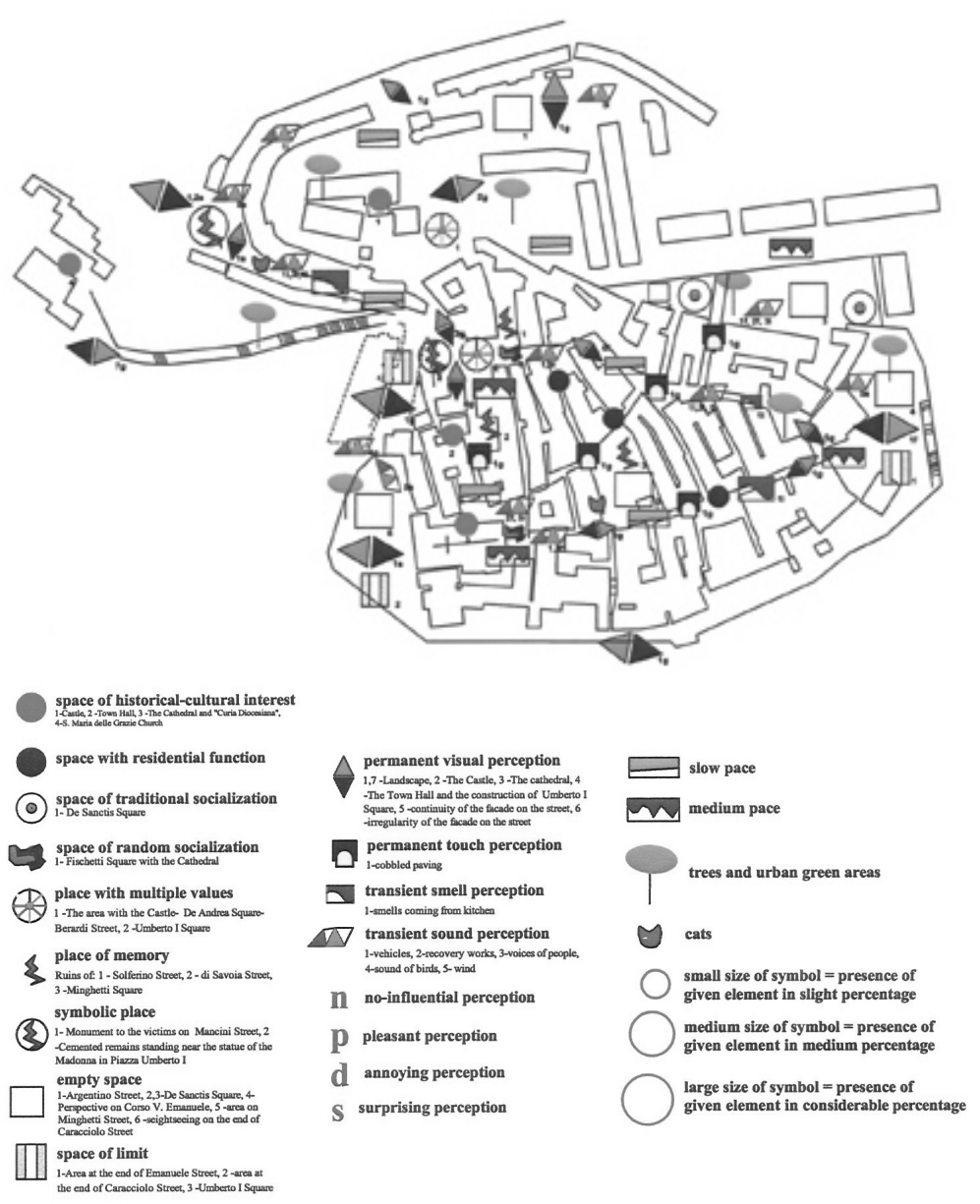

Figure 1: Sant'Angelo dei Lombardi complex map and legend. 
142 The Sustainable City IV: Urban Regeneration and Sustainability

Table 1: Symbols used on the map with corresponding weighting and features.

\begin{tabular}{|c|c|c|}
\hline Element & Weighting & Features \\
\hline \multirow{3}{*}{ Place of historical-cultural interest } & \multirow{3}{*}{5} & State of conservation \\
\hline & & Degree of contextualisation \\
\hline & & Quantitative presence \\
\hline \multirow{3}{*}{ Place with residential function } & \multirow{3}{*}{5} & State of conservation \\
\hline & & Degree of use \\
\hline & & Quantitative presence \\
\hline \multirow{3}{*}{ Place of traditional socialization } & \multirow{3}{*}{5} & Degree of contextualisation \\
\hline & & Degree of use \\
\hline & & Quantitative presence \\
\hline \multirow[t]{3}{*}{ Place of random socialization } & \multirow{3}{*}{3} & State of conservation \\
\hline & & Degree of contextualisation \\
\hline & & Quantitative presence \\
\hline \multirow[t]{3}{*}{ Place of multiple values } & \multirow{3}{*}{5} & Value of single elements \\
\hline & & Degree of use \\
\hline & & Quantitative presence \\
\hline \multirow{3}{*}{ Place of memory } & \multirow{3}{*}{5} & State of conservation \\
\hline & & Degree of capacity to recall the event \\
\hline & & Quantitative presence \\
\hline \multirow{4}{*}{ Symbolic place } & \multirow{4}{*}{5} & Degree of contextualisation \\
\hline & & Degree of capacity to recall the event \\
\hline & & Degree of use \\
\hline & & Quantitative presence \\
\hline \multirow[t]{3}{*}{ Empty space } & \multirow{3}{*}{2} & Degree of contextualisation \\
\hline & & Value of single elements \\
\hline & & Quantitative presence \\
\hline \multirow[t]{3}{*}{ Space of limit } & \multirow{3}{*}{2} & Degree of contextualisation \\
\hline & & Value of single elements \\
\hline & & Quantitative presence \\
\hline \multirow[t]{2}{*}{ Permanent visual perception } & \multirow{2}{*}{3} & Degree of perception \\
\hline & & Quantitative presence \\
\hline \multirow{2}{*}{ Permanent touch perception } & \multirow{2}{*}{2} & Degree of perception \\
\hline & & Quantitative presence \\
\hline \multirow[t]{2}{*}{ Transient smell perception } & \multirow{2}{*}{1} & Degree of perception \\
\hline & & Quantitative presence \\
\hline \multirow[t]{2}{*}{ Transient sound perception } & 3 & Degree of perception \\
\hline & & Quantitative presence \\
\hline Slow pace & 2 & Degree of perception \\
\hline & & Quantitative presence \\
\hline Medium pace & 2 & Degree of perception \\
\hline & & Quantitative presence \\
\hline Trees and urban green area & & Degree of perception \\
\hline & 4 & State of maintenance \\
\hline & & Quantitative presence \\
\hline Animals & 2 & Degree of perception \\
\hline & & Quantitative presence \\
\hline
\end{tabular}


On the contrary it is much more incumbent than appears at first sight in the enormous, untreated gaps in the townscape in Piazza Umberto, Via Caracciolo and Corso $V$. Emanuele, where one senses that no choices have been made, leaving these places without any sense of identity. In general there are no specifically tourist venues, although there are postcards on sale in some shops, and some notices indicating an itinerary featuring the historical monuments; there are very few restaurants or venues for young people. The elements used in calculating the sustainable place-identity index all feature on the map, see table 1. The absolute index of the historical centre of Sant'Angelo comes out as 551, while the median index comes out as 9.8. On the basis of the elements taken into consideration, values of the absolute index between 174 and 406 indicate acceptable place-identity sustainability; from 406 to 638 reasonable sustainability, and from 632 to 870 maximal place-identity sustainability. As for the median index, the value for acceptable place-identity sustainability ranges from 3.1 to 7.2, reasonable sustainability from 7.2 to 11.3 , and maximal sustainability from 11.3 to 15.5 . Thus the identity of Sant'Angelo is found to rank as reasonable, both on the absolute index, in terms of the quantity of characterising elements, and on the median index, in terms of the quality of the elements assessed. The reconstruction of the historical centre took into account the identity of the places, and managed not to destroy it. Even now, however, the centre of Sant'Angelo dei Lombardi has still not really come back to life, and in some spots the silence is so pervasive as to block out any other perception. Few services or activities are carried on in this centre, and there are few residents. The memory of the earthquake still haunts in this site, which appears to be frozen in time in spite of the reconstruction. What remains to be done in the way of reconstruction could take into account this index in planning the interventions, in order to enhance the cultural assets that do exist and ensure greater involvement of the population.

\subsection{The sustainable place-identity index of Kitano - Cho}

The area of Kitano-Cho is situated on a hillside. It begins from the junction of Kitanozaka Avenue and Kitano Street, the area's main trunk road, with side streets and paths leading up Mount Rokko, which delimits the study area. Few streets bear names, and although the houses are numbered, they are rarely specified. The quarter features several museum houses, all recently rebuilt. There are also various residential buildings, varying in typology and height but all made of reinforced concrete in a rational style devoid of decoration, and temples and shrines belonging to different religious cults. It is undoubtedly significant that the residential buildings and museum houses exist alongside the temples and shrines. The area is densely populated, with a youth hostel, and there is no sense of this being an empty or deserted quarter. The most interesting museum houses are situated in the eastern zone. There are also some contemporary buildings featuring high quality architecture along the mountainside and on Kitano Street. The buildings in the western zone are less interesting, and there are some overgrown plots. One sees few of the elements denoting globalisation, especially in comparison with the rest of the city: some 
satellite dishes on houses, the typical Japanese drinks vending machines and the ungainly electricity pylons lining the whole route are perhaps the most striking features. The urban décor has no particular interest; the most distinctive features are the manhole covers bearing images of the city of Kobe, such as the tower, the harbour, and so on. Kitano Cho Plaza is the area's nerve centre, much photographed by tourists: it is a round open space, with ramps for people to sit on, although most visitors just pause to take photographs. From here one can see the various places in Kitano-Cho: Rheinen House, sculptures featuring jazz musicians, and the cluster of skyscrapers in the other zone of the city. This curious area is much visited by Japanese tourists and also used for weddings. In fact there are several boutiques for wedding dresses and some isolated properties where weddings and receptions are organized. Remains of the earthquake are only to be seen in an area of vegetation beneath the House of Holland, where amongst the trees one can glimpse a devastated house. The itinerary runs downhill, and some stretches are so steep that they provide the most striking tactile perceptions. Almost all the streets running perpendicular to Kitano-Cho Street are made up and carry one-way traffic, breaking up the visual perception. The streets and paths further up are not sloping and are narrower with more vegetation. They are not made up, and ensure a more harmonious visual perception. At the highest point one's attention is held by the mountain, and low down by skyscrapers in the modern zone of the city. The most notable acoustic perception concerns the silence. Passing tourists, the noise of the fountain and of jazz from Kitano-Cho Plaza, the cries of crows and passing cars are the only sounds which occasionally interrupt the silence. The most significant smell is green tea, used by the shopkeepers to make ice cream and butter, and featuring copiously in local cooking. Some kiosks early on in the itinerary contribute to this particular perception. The pace of life is invariably tranquil, except for Kitano Street where it is moderate and at times busy. Few of those approached took any interest in the interview, apart from the owners of the museum houses. Not a single monument in the area was considered to be particularly significant, apart from the museum houses. The reconstruction in fact gave special importance to these features as being a source of revenue from tourists. The memory of the earthquake is still alive, but there are no significant mementoes in the area. In the eastern zone there is a station of Scinkanzen, a metro station and a cablecar terminus. The elements used in calculating the sustainable placeidentity index all feature on the map, as shown in table 1. The absolute index of the historical centre of Kitano-Cho comes out as 1342, while the median index is 11.5. On the basis of the elements taken into consideration, values of the absolute index ranging from 396 to 924 indicate acceptable place-identity sustainability; from 924 to 1452 reasonable sustainability, and from 1452 to 1980 maximal place-identity sustainability. As for the median index, the value for acceptable place-identity sustainability ranges from 3.4 to 7.9 , reasonable sustainability from 7.9 to 12.4 , and maximal sustainability from 12.4 to 16.9 . Thus the identity of Kitano-Cho, like that of Sant'Angelo, is found to rank as reasonable, both on the absolute index, in terms of the quantity of characterising elements, and on the median index, in terms of the quality of the elements 
assessed. This is probably due to the contrast between the eastern area, with the majority of the museum houses, and the western area, where there are still some overgrown plots and buildings under construction. Although the destination is mixed, tourist and residential, the area appears to have been designed for tourism, and can have a rather "quaint" atmosphere. The post-earthquake reconstruction concentrated above all on restoring the museum houses as the main source of income for the local inhabitants. In completing the reconstruction this index could cause planners to consider how to integrate the cultural patrimony in the area and make other sites more attractive, not least for the benefit of the local residents.

\subsection{Observations}

The sustainable place-identity index has been calculated for different contexts, with quite satisfactory results. Nonetheless the construction of complex indices for studying sustainability is still at an experimental stage. In particular, the index we have presented has only been verified in theory, albeit using case studies. It is one thing to compare index values for different places, and another thing to compare the different indices for a single place. As can easily be imagined, each place is characterised by particular features which distinguish it from other places. In order to be able to compare two different places, these features have to be assimilated in categories. This is a delicate operation requiring great care and attention if the specific quality of a place is not to be sacrificed. In the two case studies we have illustrated, we believe that the assimilation of features in more general categories has not compromised the end result. Another delicate issue concerns the weighting to be given to each feature. We found that this could in fact change according to the area under analysis: a certain perception, for example, cannot always carry the same weight for every place. In attributing the same weight to the same type of symbol regardless of place, one is in fact introducing an approximation. We also found, during other experiments, that the weighting can change according to the index being calculated, and hence it would probably be more correct to speak of relative weight. In any case we believe it is appropriate to calculate the index based on other data obtained using the PlaceMaker, and to try out the method for a sufficiently high number of cases to be able to establish a scale of values that will provide an object evaluation of the results. The first experiment in using the indices for sustainable planning showed that it is the tools for the evaluation of urban impact and territorial control at the urban level which are the most suitable.

\section{Conclusions}

We have presented the sustainable place-identity index and its use in supporting the study and planning of sustainable urban landscapes. This has involved looking at case studies of historical centres in two very different geographical locations and cultures, one in Italy and the other in Japan. They had in common 
some territorial features and the fact of being destroyed by a violent earthquake, causing a large number of casualties and widespread damage to both buildings and the identity of the two places. The method of analysis used to identify the characteristic features in place identity was the PlaceMaker. The absolute index of the historical centre of Sant'Angelo came out as 551, and the median index 9.8. For the historical centre of Kitano-Cho, the corresponding values were 1342 and 11.5. Thus both cases were found to rank as reasonable, both on the absolute index, in terms of the quantity of characterising features, and on the median index, in terms of the quality of the features assessed. Furthermore there was a good match between the quantity and quality of the features indicating the place's sustainability. What remains to be done in the way of reconstruction should take into account these indices in planning sustainable interventions enhancing the cultural assets that do exist and ensure greater involvement of the population, in the case of Sant'Angelo, and how to integrate the cultural patrimony in the area and make other sites more attractive, not least for the benefit of the local residents in the case of Kitano-Cho. If there had been scarce correlation between the two indices, this would have meant either many representative features with a low sustainability or few features able to determine a high level of sustainability. In such cases what remains to be done in the way of reconstruction should also seek to redress the balance between the qualitative and quantitative aspects of place identity sustainability. The observations on the method used in calculating the sustainable place-identity index concerned in particular the criteria for establishing the weighting and the correlation of places with different specific elements. The prime users of these indicators are likely to be urban planners, administrators, citizens, and all those involved in sustainable

urban construction. In this respect we feel that the tools for the evaluation of urban impact and territorial control at the urban level which are the most suitable for using the indices in planning sustainable urban landscapes.

\section{References}

[1] Nijkamp, P., Perrels, A.H., Sustainable Cities in Europe, Earthscan: London, 1994.

[2] Cecchini, A. Fulici, F. La valutazione di impatto urbano. Una proposta metodologica, FrancoAngeli: Milano, 1994.

[3] European Conference on Sustainable Cities and Towns, Aalborg Charter, Denmark, 27 May 1994.

[4] Sepe, M. The PlaceMaker: a flexible and interactive tool to support the sustainable city construction and transformation, Proc. of Sustainable Planning 2005, WIT Press: Southampton, 2005 (Pagg.1525-1534). 